Review

\title{
The Laboratory Diagnosis of Clostridioides difficile Infection: An update of current laboratory practice
}

\author{
Ali Mohammed Somily ${ }^{1}$, Mohammad Aatif Khan², Muhammad Morshed ${ }^{3}$ \\ ${ }^{1}$ Department of Pathology and Laboratory Medicine, College of Medicine, King Saud University, King Saud \\ University Medical City, Riyadh, Saudi Arabia \\ ${ }^{2}$ Microbiology Laboratory, Prince Mohammad Bin Abdul Aziz Hospital, Riyadh, Saudi Arabia \\ ${ }^{3}$ Department of Pathology and Laboratory Medicine, University of British Columbia, Vancouver, BC, Canada; \\ British Columbia Centre for Disease Control Public Health Laboratory, Vancouver, Canada
}

\begin{abstract}
Clostridioides difficile can cause colitis and is associated with hospital acquired infections. The $C$. difficile infection (CDI) is due to production of toxins A and B which bind to epithelial cell surface receptors and triggers signaling pathways, leading to loss of epithelial barrier function, apoptosis, and inflammation, culminating in diarrheal disease. In early days, laboratory diagnosis of CDI was based on cell culture, identification of toxins, and their cytopathic effects. These assays were replaced by enzyme immunoassays for the detection of $C$. difficile toxins and the GDH house-keeping gene for improved specificity. Later, molecular assays with higher sensitivity were introduced which are becoming easier to incorporate into the test algorithm. The diagnosis of CDI and significance of laboratory results can be challenging with asymptomatic colonization of $C$. difficile in some patients. Test result interpretation is even more challenging due to multiple guidelines, emerging resistant $C$. difficile ribotypes, as well as differences in disease prevalence. An accurate test result for diagnosis of CDI depends on selecting patients with high pre-test probability, collecting an acceptable stool specimen, and a thorough understanding of current test methods.
\end{abstract}

Key words: Clostridiodes difficile; Clostridium; Laboratory diagnosis; update; practice.

J Infect Dev Ctries 2021; 15(10):1364-1375. doi:10.3855/jidc.13217

(Received 07 June 2020 - Accepted 27 June 2021)

Copyright (C) 2021 Somily et al. This is an open-access article distributed under the Creative Commons Attribution License, which permits unrestricted use, distribution, and reproduction in any medium, provided the original work is properly cited.

\section{Introduction}

Clostridioides difficile (formerly known as Clostridium difficile) is an anaerobic, spore-forming Gram-positive bacillus responsible for toxin-mediated gastrointestinal disease, ranging from mild to life threatening infection in some patients. This organism has been recently re-classified as Clostridioides difficile which was based on phenotypic, chemotaxonomic, and phylogenetic analysis [1]. C. difficile infection (CDI) is often associated with previous episodes of antibiotic treatment and is an important cause of health care associated infections (HAI). While CDI is considered a serious disease, asymptomatic carriage of toxigenic and non-toxigenic $C$. difficile is commonly found in hospitalized patients and the community.

The use of broad-spectrum antibiotics contributes to CDI by disrupting intestinal commensal flora. This allows spores of $C$. difficile to colonize and trigger gastrointestinal infection. Subsequently, $C$. difficile's elaborate toxins lead to varying degrees of inflammation and colitis. Since CDI is considered an important HAI, its surveillance, clinical diagnosis, laboratory detection of pathogen with toxins, infection rates and reporting are important elements of quality of health care for any hospital administration. Concerned health regulatory authorities should provide an acceptable standard of care where CDI rates are minimal and do not complicate an admitted patient's length of stay and clinical outcomes. In some countries, CDI rates are reported routinely to health regulatory authorities and are closely monitored as a key performance indicator of the institution. This review article will focus on CDI diagnosis and emphasize the current practice in laboratory diagnosis and the preanalytical issues related to quality of specimen that influence the pre-test probability of CDI. In addition, a review of new technologies, advancements in analytical methods, and their post-analytical value in CDI diagnosis will be included as well.

In a recent multi-state point-prevalence survey in USA hospitals, CDI ranked as the most common infectious agent related to HAIs. In the last decade, the 
incidence and morbidity due to CDI have significantly increased in multi-state US hospitals [2], as well as disease mortality [3]. The reasons for the rise reported in the literature are multifactorial, however a number of contributory factors have been identified. These include but are not limited to an aging population that is susceptible to CDI with poorer outcomes, the emergence of two hypervirulent ribotypes, 027 and 078, and improvements in diagnostic tests with better detection of the toxin genes and their products.

Risk factors for CDI in elderly patients include increased antibiotic use, prolonged hospitalization, and chemotherapy $[4,5]$. The epidemiology of $C$. difficile infection changed dramatically in early 2000 s, when a new hypervirulent strain of $C$. difficile, the NAP1/BI/027, was identified and found to be responsible for several outbreaks in North America [6]. Since then, several pathogen and host factors have been identified that potentially play a major role in the pathogenesis and severity of CDI [6]. These studies have improved our understanding of why exposure to antimicrobial treatment is perhaps the most important risk factor in CDI. The pivotal role of gastrointestinal microbiome in preventing CDI has increasingly becoming clear in recent studies. One such study highlighted how the loss of microbiome can create an environment that promotes growth of $C$. difficile and stimulates the production of toxins [7]. This study suggests that a possible mechanism in which the microbiome can prevent infection is the conversion of primary bile salts to their secondary forms which inhibits toxin-producing vegetative cells of $C$. difficile and prevent their multiplication. A loss of microbiome leads to an accumulation of primary bile salts which can stimulate germination of $C$. difficile spores. When the microbiome is diminished by antimicrobials, $C$. difficile spore formation, growth of the organism, and toxin production is enhanced in colonized persons [7].

Recent studies have also identified certain species of intestinal microbiome that may play an important role in protecting patients against CDI. These protective organisms include but are not limited to Bacteroides, Ruminococcaceae, Lachnospiraceae, and Eubacterium. $[7,8]$. The absence or loss of these species following antibiotic therapy creates an environment in which toxigenic $C$. difficile can propagate and cause disease [9]. In patients with recurrent CDI, re-introducing these protective organisms in fecal microbiota transplants led to resolution of disease in $90 \%$ of patients compared to resolution of only $30 \%$ of patients receiving $C$. difficile specific antimicrobial therapy [10-12]. Taken together, the above findings reiterate the role of antibiotic therapy as a key risk factor for CDI as well as the importance of re-constituting the protective gut microbiota in patients with CDI.

To date, an increased understanding of microbiome has provided novel opportunities for therapeutics of CDI, however, the optimal laboratory diagnosis of CDI remains elusive. This is in spite of advancements in molecular technologies and improvements in the performance of immunological methods to detect $C$. difficile enzymes and toxins. This review will attempt to summarize advanced diagnostic tests currently available for CDI so as to better understand this toxinmediated disease.

Several US Food and Drug Administration (FDA)approved tests are commercially available to detect the presence of $C$. difficile in stool samples. These tests vary in technical complexity, cost effectiveness, sensitivity, specificity, and positive and negative predictive values (PPV, NPV). Our aim in this review is to elaborate on both new and old test methods and provide an update on recommended laboratory test algorithms.

It is important for clinicians to know what constitutes an acceptable specimen for laboratory tests and which patients should be investigated for the diagnosis of CDI. Patients with a high pre-test probability who should be tested are those currently receiving antibiotics or have received antibiotics in the past eight weeks. These should have a minimum of three documented episodes of liquid diarrhea stool per day [13]. CDI is most prevalent in elderly patients (more than 65 years of age), specifically those who have recently been admitted into hospital and those living in long-term care facilities [6]. A useful guide for an acceptable stool specimen is one that takes the shape of the container after inverting the vessel. Specimens should not be collected from patients that have received laxatives in the last 48 hours as this not considered acceptable for testing [6].

\section{Laboratory methods for detection of C. difficile infection}

A case definition of CDI requires a clinical diagnosis supported by laboratory findings in an acceptable time frame for early isolation, initiation of specific antimicrobial therapy, and prevention of further transmission. Historically, the culture-based diagnosis of $C$. difficile has been difficult since it requires specific selective media and growth conditions. The long turn-around time for culture-based identification further limits its practical usefulness and diagnostic value in a clinical laboratory. However, tests 
known as cell culture cytotoxicity neutralization assay (CNA) and toxigenic culture (TC) are considered as gold standard reference tests for detection of $C$. difficile. These tests require multiple steps, expertise in mammalian cell cultures, and are also time consuming to perform. Furthermore, these tests are available mostly in reference and research laboratories. Later, rapid enzyme immunoassays (EIA) for the detection of C. difficile were developed, became commercially available, widely implemented in community laboratories, and incorporated into diagnostic algorithms. The EIA test is simple, cost-effective, and rapid; however, some studies have shown that its analytical sensitivity is low compared to the gold standard CNA and TC for detecting $C$. difficile toxins [6]. This has led to a search for a better test that is more sensitive and specific; subsequently, the molecular method known as the Nucleic Acid Amplification test (NAAT), which detects $C$. difficile toxin genes in stool specimens, was introduced and approved as a diagnostic test by FDA and other health regulatory agencies [6]. Further details about the NAAT will be described elsewhere in this article.

\section{Cytotoxicity Neutralization Assay (CNA)}

In this test, a stool filtrate is applied to monolayer of epithelial cell line grown in culture. The type of cell line employed includes human foreskin fibroblasts, human diploid fibroblast, MRC5 lung fibroblast, McCoy cells, Vero cells, as well as Hep-2 cells. After 24 to 48 hours of incubation, cells are observed for lysis as a result of cytopathic effect (CPE) of $C$. difficile toxins present in stools. If CPE is observed, a neutralization assay is performed to demonstrate that CPE is mediated specifically by C. difficile toxin and not due to a non-specific toxicity. The neutralization step is performed using $C$. sordelli or $C$. difficile antiserum which inhibits toxin mediated effects on cell viability. CNA primarily detects toxin B; however, toxin A may also be detected [14]. The sensitivity of CNA ranges from $65 \%-90 \%$ [6], whereas TC sensitivity ranges from $75 \%$ to $85 \%$ [6]. The performance of CNA is influenced by a number of factors including degradation of toxin due to delays in specimen transport that diminishes its cytotoxicity, types of cell line used, the time between symptom onset and performance of the assay, and finally the effect of $C$. difficile specific antibiotic therapy [15]. As a result, considering CNA as the reference method has become questionable; although, this test has historically been considered as the gold standard assay [16].
Toxigenic Culture (TC)

Toxigenic culture (TC) is a culture based method which involves isolation of the $C$. difficile from fecal specimens and determining if the specific bacterial strain produces toxins. While concensus is lacking, a number of variations in this method have been described that are based on using anaerobic agar and broth culture with selective and differential agents $[17,18]$. Selective agents inhibit the normal fecal flora while enhancing the recovery of $C$. difficile. A number of growth media have been utilized in TC which depend on the ability of $C$. difficile to ferment fructose $[17,18]$.

Growth medium that contains cycloserine, cefoxitin, and fructose agar (CCFA) is a selective and differential media that can isolate C.difficile $[17,18]$. A number of modifications of this media have been reported including cefoxitin, cycloserine, and egg yolk agar (CCEY), otherwise referred to as Brazier's medium. Another modification is the cefoxitin, cycloserine, egg yolk agar with lysozyme (CCEYL) $[17,18]$. Addition of lysozyme and taurocholate in the growth medium was found to increase the production of C. difficile vegetative spores [17]. The media should be pre-reduced prior to inoculation of the specimen to enhance recovery and isolation of $C$. difficile, then incubated for at least 48 hours and held for up to five days before being reported as negative [17]. Hink et al. observed that the most sensitive method for isolating $C$. difficile from stool specimens was to heat-shock the specimen at $80^{\circ} \mathrm{C}$ before inoculating the CCFA mannitol broth containing taurocholate and lysozyme [17].

Colonies suspicious of $C$. difficile are yellowish to white, circular to irregular with a rhizoid or jagged edge, and have a typical ground class appearance. $C$. difficile colonies have a special horse-barn odor due to the production of para-cresol and exhibit yellow-green fluorescence under UV light. Culture based identification is based on colony morphology, gram stain, typical odor, and biochemical tests such as spot indole and detection of L-proline-naphthylamide. Commercial biochemical kits such as RapidANA (Remel, Lenexa, KS, USA) can be used for presumptive identification of $C$. difficile in addition to MALDI-TOF instrument, which can identify suspicious colonies from selective agar [6].

After identification of $C$. difficile, isolates should be subjected to further tests to demonstrate its toxin production. Before commercial systems became available, this could be achieved by performing the CNA assay. Recently, however, $C$. difficile toxin EIA can detect toxin production in cultured isolates. A 
newly developed selective CHROMagar is a rapid and accurate method for detection of $C$. difficile directly from stool sample [17]. Colonies of $C$. difficile appear as black with a clear background. However, toxin production needs to be ascertained further by another method as indicated above. Currently, TC is considered the gold standard for isolating $C$. difficile in fecal specimens and the Society for Health Care Epidemiology of America/Infectious Disease Society of America (SHEA/IDSA) guidelines recommend the use of this method for method comparison studies [15].

\section{C. difficile Toxin Enzyme Immunoassays (EIA)}

Enzyme Immunoassay (EIA) was first described by Lyerly et al. in 1998 for the detection of $C$. difficile toxin A in culture supernatants [19]. Since then, this test has improved significantly and is currently based on monoclonal and polyclonal antibody detection of toxins. For several years, toxin EIA was the most frequently used test in the laboratory diagnosis of CDI due to its simplicity, low cost, and rapid turn-around time. Initially, toxin EIA only detected toxin A, however, toxin A-negative toxin B positive disease was increasingly recognized to cause CDI in several countries [6]. It is now recommended that EIA should detect both toxins A and B in clinical samples as both are thought to be important in the pathogenesis of CDI [20]. Currently, there are a number of commercially available EIAs that detect both $C$. difficile toxin A and $\mathrm{B}$, based on rapid immuno-chromatographic/lateral flow assay method as well as microwell and solid phase platforms. While most toxin EIAs have excellent specificity, their sensitivity varies widely from $40 \%$ to $100 \%$ [21,22]. Further studies have shown that some toxin EIAs have a low PPV in diagnosing CDI [23]. Due to limitations in analytical sensitivity, the $C$. difficile toxin EIAs as stand-alone tests are not considered an acceptable method for diagnosing CDI $[24,25]$ and the SHEA/IDSA guidelines recommended that the use of toxin EIA alone is not sufficient in the diagnosis of CDI.

\section{Glutamate dehydrogenase (GDH)}

Glutamate dehydrogenase (GDH) is an enzyme that is produced in $C$. difficile and involved in bacterial metabolic pathways. GDH is considered a housekeeping enzyme and thus produced in both toxigenic and non-toxigenic strains of $C$. difficile. The GDH EIA is therefore an ideal screening test for CDI in fecal specimens. However, a confirmatory test is needed and either toxin EIA or molecular test can be used for detecting toxin genes, as described below
$[21,26]$. GDH is therefore an attractive screening test to quickly rule out CDI in laboratories that do not have expertise and have not implemented a molecular confirmatory test. However, the reliability of GDH EIA in ruling out CDI is still unproven. A study by Tenover et al. in 2010 observed that the sensitivity of GDH EIA can vary depending on strain type. In particular, this study found a reduced sensitivity for ribotypes 002 , 007, and 106 in GDH EIA [27].

Another large study conducted in 2012 evaluated 104 isolates of $C$. difficile that contained 77 ribotypes including toxigenic and non-toxigenic fecal strains collected from several countries during the period of 2004 to 2011 [28]. This study showed that all strains studied carried the $g l u D$ gene, which encodes for GDH, and the predicted amino acid sequences of most isolates were also identical. Furthermore, all strains produced GDH and were reliably detected by a number of commercial EIAs including C. Diff Quik Chek ${ }^{\circledR}$, C. Diff Chek-60 ${ }^{\circledR}$ and C. Diff Quik Chek Complete ${ }^{\circledR}$ (TECHLAB, Blacksburg, VA, USA). Another study by Goldenberg et al. demonstrated that the GDH detection by C. Diff Chek- $60^{\circledR}$ did not vary significantly among 64 isolates of $C$. difficile that were grouped into 002, 005, 023, 027, and 078 ribotypes [29]. Collectively, these findings suggest that the performance of commercial GDH EIA is not dependent on strains or ribotypes of $C$. difficile and may be useful when included as part of a test algorithm.

\section{Combined GDH and toxin EIA}

A number of commercially available EIAs combine the detection of $C$. difficile GDH with toxin $\mathrm{A}$ and $\mathrm{B}$ as a single test. These rapid tests are also simple to perform and cost effective compared to gold standard and molecular methods. The sensitivity of GDH detection is comparable to stand-alone GDH EIA, however, the toxin component suffers from the same lack of sensitivity as stand-alone toxin EIA mentioned earlier [30] and when compared to GeneXpert $C$. difficile PCR assay [31]. When using these combined assays, samples that are GDH and toxin negative can be reported as negative. Similarly, samples that are GDH and toxin positive can be reported as positive. However, GDH positive and toxin negative should be subjected to further $C$. difficile molecular testing or CNA/TC tests to rule out CDI [32].

\section{Molecular tests for clinical uses \\ Polymerase Chain Reaction (PCR)}

Studies of molecular assays based on nucleic acid amplification for the detection of $C$. difficile in fecal 
samples were published in the early 1990s [33-35]. These were conventional PCR assays that targeted the $t c d A$ gene which encodes toxin $\mathrm{A}, t c d B$ genes which encodes toxin $\mathrm{B}$, and $16 \mathrm{~s}$ rRNA. The procedure involved manual extraction of nucleic acid by phenolchloroform and multiple preparation steps to run the assay. PCR products were detected by gel electrophoresis and Southern blot analysis. In spite of some cross reactivity with other clostridial species, manually designed primers were able to amplify intended gene targets with better sensitivity than anaerobic culture-based tests and CNA [35]. These findings were early indicators that nucleic acid amplification tests (NAAT) could be a better platform for the accurate and timely detection of $C$. difficile.

This next advancement in the field of molecular biology came with improvements in extraction of nucleic acid using spin columns and commercially available reagents which simplified the procedure for PCR. The advent of real-time PCR (RT-PCR) was a breakthrough in diagnostic microbiology; this assay utilized fluorescent primer and probes to detect specific sequences during amplification of target genes. Instruments known as thermal cyclers used for PCR have developed even further with smaller footprint and benchtop models linked to computerized software that displays amplification curves during the procedure. Importantly, these systems have become more automated, more cost effective and affordable for small and medium sized diagnostic laboratories, and require less expertise to operate and troubleshoot compared to the earlier large and cumbersome systems.

\section{Nucleic Acid Amplification tests (NAAT)}

Currently, NAAT based on RT-PCR instruments include the Roche LightCycler (Pleasanton, CA, USA), Cepheid GeneXpert (Sunnyvale, CA, USA), and the iCycler IQ (Bio-Rad Inc., CA, USA) to name a few. The first FDA cleared molecular assays for the detection of C. difficile in fecal samples became available commercially in 2009. This was the BD GeneOhm Cdiff ${ }^{\circledR}$ assay (BD Diagnostics, MD, USA) which detects the conserved regions of $t c d B$ with detection of amplified products based on fluorogenic hybridization probes known as molecular beacons. The current version of this platform is the BD Max $\operatorname{Cdiff}^{\circledR}$ which is fully automated in nucleic acid extraction, amplification, and detection, and significantly reduces the set-up time. This platform received FDA clearance in 2013 for the detection of C. difficile in stool samples [36].
The Cepheid Xpert C. difficile ${ }^{\circledR} \mathrm{PCR}$ assay (Cepheid, CA, USA) is also a fully automated, integrated, closed system that detects not only $t c d B$ but also the $c d t$ binary toxin genes, as well as the deletion at nucleotide 117 on $t c d C$ which serves as a marker for the identification of a virulent strain known as 027/NAP1/BI. A major characteristic of the Cepheid Xpert $C$. difficile PCR is that it is one of the simplest systems to use with minimum time for hands-on sample preparation and also one of the most rapid assays with a turn-around time of about only 60 minutes. This system uses disposable unitary cartridges that contain all the reagents and reaction steps necessary for realtime PCR [37]. In a two-year study conducted in UK hospitals to determine the acceptability, ease of use, lower TAT, and clinical utility of the Cepheid Xpert $C$. difficile PCR assay as a point of care test, it was found to be feasible and acceptable by nursing and technical staff in the wards and ICU [38].

In addition to the above mentioned single-plex platforms, other FDA cleared NAAT that have demonstrated superior performance in detecting $C$. difficile in stool samples include IMDx $C$. difficile for Abbott m2000 assay [39]; Verigene $C$. difficile nucleic acid test [40]; Simplexa $C$. difficile Universal Direct Real Time PCR [41]; AmpliVue C. difficile assay [41]; Illumigene $C$. difficile assay [42]; BD GeneOhm Cdiff assay [42], and; ProGastro CD assay [30].

The loop mediated isothermal amplification method known as LAMP is based on hybridization of (six primer sequences that are complementary to eight regions of target DNA sequence thereby significantly increasing the specificity of the assay $[43,44]$. In LAMP, the amplification reaction occurs under isothermal conditions and with the presence of multiple priming sites for DNA synthesis. Results are available earlier compared to conventional PCR or qPCR as the assay does not require a thermal cycling reaction and is instead based on loop amplification [43]. The Alethia Meridian Bioscience-Illumigene $C$. difficile DNA amplification assay (Cincinnati, OH, USA) is based on LAMP technology that detects the pathogenicity locus (PaLoc) of toxigenic C. difficile. The PaLoc gene segment is present in all known toxigenic strains of $C$. difficile and codes for both toxin $\mathrm{A}$ and toxin $\mathrm{B}$ genes involved in the pathogenesis of CDI. While most commercial NAAT assays described above detect the $t c d B$, the Meridian Bioscience-Illumigene $C$. difficile assay detects the $t c d A$ which encodes for toxin A. The $t c d A$ partial DNA fragment that is detected is present in all known $\mathrm{A}+\mathrm{B}+$ and $\mathrm{A}-\mathrm{B}+$ toxinotypes of $C$. difficile. Toxinotyping has become a standard method for 
studying the prevalence of $C$. difficile in different regions worldwide. Toxinotyping involves the use of PCR ribotyping, pulsed field gel electrophoresis, and multilocus gene sequencing among others. Based on restriction fragment length polymorphism, Rupnick et al. have described $C$. difficile toxinotypes as being 0 to XXVIII [45]. While toxinotype 0 is most common worldwide, other toxinotypes have been further described in developing regions [46].

The role of $C$. difficile toxins in CDI has been extensively investigated [20]; it is now known that CDI is due to three major toxins. The Rho-glycosylating toxin A, toxin B are mainly responsible for diarrhea and colitis while $5-30 \%$ of clinical isolates also produce a binary ADP-ribosylating toxin called CDT, which alters epithelial cell actin fibers [47]. However the role of CDT in pathogenesis is not clear. CDT has gained increasing importance because it is frequently produced by a hypervirulent strain of $C$. difficile known as NAP1/B1/027 (North American PFGE Type-1, Restriction Endonuclease-REA B1 and PCR ribotype027) [47]. As indicated above, most FDA-cleared NAAT target the $t c d B$, while a few target $t c d A$. However, the Cepheid GenXpert targets the $t c d B, c d t A$, and $t c d C$ nucleotide 117 deletion commonly seen in the hypervirulent NAP1/B1/027 strain [37]. In addition, the multiplex PCR platform Luminex-Nanosphere Verigene assay (Austin, Texas, USA) also detects $t c d A$, $t c d B$, and $c d t$ nucleotide 117 deletion [40].

Currently, there are two US FDA-cleared syndromic multiplex assays that can detect a panel of enteric pathogens as well as $C$. difficile toxins. These include the Luminex xTAG GPP (Luminex Corporation, USA) and Biofire FilmArray GI Panel (Biofire Diagnostics, Salt Lake, USA). In addition, there are a number of non-FDA, CE marked multiplex assays that can detect $C$. difficile toxins in stool specimens. Notable amongst these are the FTD bacterial gastroenteritis panel (Fast-Track Diagnostics, Siemens) and the Seegene Allplex GI panel which was found to be comparable in performance characteristics to the Luminex xTAG GI panel [48]. Further, the Seegene Seeplex ACE panel was reported to have similar sensitivity, specificity, PPV, and NPV to the BD GeneOhm Cdiff assay in the detection of $C$. difficile toxin B in stool specimens [49].

The application of NAAT as a potential point-ofcare test to detect $C$. difficile toxins has been recently investigated. This may be particularly useful in emergency rooms, outbreak settings, resource-limited hospitals, and long-term care facilities. Early and accurate detection of $C$. difficile toxins can allow timely clinical management with emergent fecal transplant therapy and rapid implementation of infection control measures [50]. Interestingly, the isothermal LAMP NAAT and PCR-based methods were the first platforms to receive Clinical Laboratory Improvement Amendments (CLIA) waived status in 2015 for the detection of Influenza A and B virus [51] and Streptococcus pyogenes [52], which can be performed in community pharmacies in USA.

Extreme point of care testing - referred to as POCT for infectious diseases - implies testing in low-resource settings, developing countries, tropical regions, and in conditions following an emergency, such as natural disasters and earthquakes. These settings require robust biomedical equipment, versatile reagents and kits, minimal hands-on time, and training of health care workers. This is essential to screen for and control diseases that have an outbreak potential and can spread quickly in vulnerable populations. One such example is the GenePOC ${ }^{\circledR}$ (Debiopharm, Lausanne, Switzerland), a novel microfluidic platform that is a rapid, robust, and reproducible NAAT developed for the diagnosis of infectious diseases [53]. The GenePOC CDiff $^{\circledR}$ is a qualitative point-of-care test that utilizes automated sample preparation and RT-PCR to detect $t c d B$ in liquid diarrhea stool samples for the diagnosis of CDI. The GenePOC $^{\circledR}$ platform received FDA approval in 2017 and Health Canada approval in 2018. While potentially attractive, POCT requires laboratory oversight for training, quality control, proficiency tests, instrument validation, and maintenance. In addition, interpretation of results and their clinical relevance require detailed review and analysis by experienced microbiologists.

\section{Molecular tests for Public Health uses PCR Ribotyping}

Ribotyping is bacterial strain typing method that is based on PCR amplification of intergenic spacer (ITS) region between the $16 \mathrm{~S}$ and $23 \mathrm{~S}$ rRNA genes. This region has several copy numbers with varied length in the $C$. difficile genome. A single primer pair can detect target sequences resulting in a pattern of bands ranging from 200-700 bp [54,55]. The bands can be resolved by polyacrylamide gel electrophoresis. While PCR ribotyping has good discriminatory power, it may not be able to differentiate between closely related $C$. difficile ribotypes such as 027, 106, and 017 [56]. Further, comparison of banding patterns between laboratories may differ if the data analysis software used for analysis is not similar. To resolve issues related to manual interpretation of banding patterns, PCRribotyping has recently been adapted to high-resolution 
capillary gel electrophoresis. Results show an improvement in reproducibility and allow for the comparison of results between laboratories [57]. In the UK and other European countries, attempts have been made to standardize ribotyping protocols and this method is now commonly used for surveillance and reporting to the $C$. difficile ribotyping network service [58]. The commonly reported ribotypes in the European union include the hypervirulent ribotype 027 , which declined in the UK from $55 \%$ in 2007-2008 to approximately $2.3 \%$ by $2012-2013$ [59]. The authors in this study also noted that approximately $88 \%$ of all 027 ribotypes were identified in Germany (43\%), Hungary (17\%), Poland (16\%) and Romania (12\%). In a more recent European multicenter, prospective, point prevalence EUCLID study, 125 different ribotypes were identified in 19 different countries based on PCRribotyping [59]. The hypervirulent ribotype 027 accounted for $22 \%$ of all CDI; other prevalent ribotypes included 001/072 and 014/020. The emerging ribotype 078, found in high numbers in calves and animals by some European countries, is a particular concern as well. While there has been no direct transmission to humans, circumstantial evidence suggests that this may have zoonotic potential to possibly cause human disease [60]. Unlike Europe, there is no centralized surveillance system established in the USA that serves as a depository for $C$. difficile isolates across the country. The US has experienced a change in the epidemiology of $C$. difficile ribotypes over the last decade. The three most common strains of $C$. difficile in both hospital and community acquired CDI were NAP1, NAP4, and NAP11, as identified by PFGE. These isolates corresponded to PCR ribotypes 027,020 , and 106, respectively [61]. While ribotype 027 is predominant in the USA, ribotype 078 was found to be an emerging strain. Unlike PCR ribotyping in European countries, US ribotypes are identified by PFGE method, which again makes comparisons of isolates difficult.

\section{Pulsed Field Gel Electrophoresis (PFGE)}

Pulsed field gel electrophoresis (PFGE) is the standard method for strain typing of $C$. difficile practiced in North America. This assay involves the digestion of genomic DNA with a restriction enzyme that cuts at rare sites within the genome, otherwise known as a "rare cutter." For C. difficile, SmaI restriction enzyme is commonly used for PFGE [62]. The DNA fragments produced after digestion are separated by gel electrophoresis, however the electrical field is repeatedly switched in three different directions, hence the term pulsed-field. This procedure allows for resolution of large DNA fragments for identification, which would not be possible with conventional gel electrophoresis. The banding pattern described afterwards are known as North American pulsed-field (NAP) to describe a specific ribotype. While there can be some degree of subjectivity, strains that are $>80 \%$ similar are considered to be of single pulsotype [62]. Many factors could affect the intra- and inter-laboratory reproducibility of PFGE that needs to be considered during sample preparation, processing, and interpretation, especially during outbreaks [63].

\section{Multilocus variable number tandem-repeat analysis (MLVA) and Multilocus sequence typing (MLST)}

Multilocus variable number tandem-repeat analysis (MLVA) is a typing method for $C$. difficile that is most commonly used in outbreak investigations. It has sufficient discriminatory power to identify and track strains linked to an outbreak and is useful in hospital settings. This method is based on the identification of multiple variable-number tandem-repeat loci (VNTR) that are dispersed through-out the loci of the $C$. difficile genome and can vary with some level of diversity to resolve phylogenetic relatedness between isolates. Amplicons produced during VNTR are resolved by gel electrophoresis and fragments are analyzed by an automated software [64]. While useful for outbreak investigations and hospital transmission events, MLVA has not been standardized and there is no agreed method or naming scheme that is consistent across laboratories. Due to this limitation, it is difficult to compare data obtained from different laboratories when investigating isolates in an outbreak setting.

Multilocus sequence typing (MLST) is based on typing up to 6-12 loci that consist of allele fragments of 300 to $600 \mathrm{bp}$ size in the $C$. difficile genome. These loci are typically house-keeping genes that are relatively conserved, but variations in sequence can be used to distinguish isolates $[65,66]$. These house-keeping genes include aroE, $d d l, d u t A, t p i, \operatorname{rec} A, g m k$, and $\operatorname{sod} A$, and were first described in 2004 by Lemee et al. for analysis of a group of $C$. difficile isolates [65]. MLST starts with PCR amplification of house-keeping genes followed by Sanger sequencing method. For many bacterial species, MLST used to be considered the gold standard for strain typing; however, due to increasing cost and longer turnaround time it is being increasingly replaced by new era, high-throughput sequencing methods such as whole genome sequencing [67]. 


\section{Whole Genome Sequencing (WGS)}

Whole genome sequencing (WGS) is potentially an ideal strain typing method which can analyze transmission events within clusters of cases and outbreak settings. WGS was initially considered too expensive and too cumbersome to perform within a time frame that is useful in real-time outbreak situations. However, a gradual decrease in cost, improvement in speed, and its high-through put capacity has since made this assay attractive for many laboratories. One major requirement is an acceptable user-friendly software that can analyze sequence data which is often produced in a large number $[68,69]$. In a recent study, WGS was used to investigate transmission patterns between infected and colonized patients at six Canadian hospitals. The NAP1/027/ST1 strain was found to be the most common strain in $62 \%$ of infected and $26 \%$ of colonized patients [70]. In another study, WGS helped to distinguish relapse cases from reinfection cases, and identified transmission events among patients with recurrent $C$. difficile infections [71]. In spite of great potential applications and recent advancements in instrumentation, some concerns remain for the mainstream implementation of WGS. These include cost effectiveness, lack of expert trained personnel, limited availability of bioinformaticians for downstream data analysis, inadequate reference microbial databases, and challenges with implementing effective, standardized, and accredited protocols [70,72]. Various platforms are available commercially and can be utilized in clinical microbiology laboratory settings, such as MinION (Oxford Nanopore Technologies, Oxford, UK), Ion Torrent (Thermo Fisher Scientific, Waltham, Massachusetts, USA), and Illumina Hiseq (Illumina, San Diego, USA), for documentation and transmission confirmation of $C$. difficile infections from a patient who is either infected or colonized to other patients.

\section{Laboratory Test Considerations and Algorithms}

Since $C$. difficile can colonize the gut asymptomatically in many adults and children, it is necessary to limit testing to only those patients with the highest pre-test probability for CDI diagnosis. These criteria are also included as guideline recommendations of various international professional organizations such as SHEA/IDSA, American Society for Microbiology (ASM), and European Society of Clinical Microbiology and Infectious Diseases (ESCMID). The patients for whom diagnosis of CDI should be considered include hospitalized patients who develop diarrhea with liquid stool passed at least three times per day. To determine the acceptability of stool specimens, laboratories should develop rejection criteria for stool specimens that are formed or those that do not take the shape of the vessel upon inverting the container. Other rejection criteria that may be included are not to test for CDI in asymptomatic patients, limit testing to patients who have been admitted to a health care facility for more than 48-72 hours, not perform a test of cure after therapy, and avoiding repeat testing [73].

As indicated above, the NAAT assay for the detection of $C$. difficile toxin is more sensitive than EIA and may be used as a stand-alone test. Alternatively, to overcome some of the limitations of assays, different tests may be combined into an algorithm to optimize the laboratory diagnosis of CDI. The best practice guidelines for laboratory tests that should be performed for CDI varies between different single stand-alone tests, a two-test algorithm, and even a three-test procedure. ASM recommends NAAT as a stand-alone test, as well as two-step GDH followed by NAAT. Both of these practices have a high overall strength of evidence compared to the two-step GDH/Toxin followed by NAAT, which was observed to have moderate overall strength of evidence. Laboratory developed tests (LDT) for the detection of toxigenic $C$. difficile offers low cost combined with equivalent performance characteristics. A comparison of LDT with a commercially available system for the detection of toxigenic $C$. difficile in multiple laboratories demonstrated similar analytical performance. However, the authors concluded that intrinsic differences based on the gene targets amplified may influence performance characteristics [74].

While some authorities recommend one stand-alone assay, the ESCMID guidelines recommended that no single test should be used as a stand-alone test due to their low PPV at low CDI prevalence rates. For optimal diagnosis of CDI, ESCMID recommends a two-step test algorithm [73]. In this scheme, the first test should have a high NPV - a highly sensitive assay that can reliably rule out CDI. This first test can be either a GDH EIA or NAAT assay. The choice between the two is dependent on cost effectiveness, volume of test performed, and available expertise. If the result is positive, it should be followed by another test with a high PPV-a highly specific assay, such as a Toxin A/B EIA. If the second test is positive, the final interpretation is a diagnosis of CDI. Patients with a negative second test for toxins should be re-evaluated for the possibility of true infection with a toxin level below the threshold for detection. Alternatively, these patients may be carriers of a new toxigenic strain of $C$. difficile. The decision to 
implement a stand-alone test or a two-step procedure will be determined by prevalence rates of CDI, cost effectiveness, and the availability of expertise and resources within a given laboratory setting.

\section{Conclusions}

The laboratory diagnosis of CDI could be challenging due to the lack of a stand-alone gold standard test that can be performed routinely in clinical laboratories. While the performance of EIA and molecular assays has improved significantly in recent years, interpretation of results obtained from different types of algorithms based on two-step and three-step assays might be challenging for physicians. The emergence of novel ribotypes that have zoonotic potential and may be food-borne, such as 078 , makes definitive diagnosis more complicated. This review describes the current test methodologies available for detecting $C$. difficile in stool specimens. The principle of each assay, its advantages and limitations, were reviewed along with the clinical utilities of the test results. It should be noted that the emergence of multiple ribotypes and better diagnostics have resulted in an increased incidence of $C$. difficile disease being reported in many regions worldwide. This increased incidence can be of concern in health care settings. Several studies have described risk factors for CDI, which include previous antibiotics, proton pump inhibitors, elderly age, and previous hospitalization. The use of the ATLAS scoring system facilitates risk stratification, prediction, and management of recurrent CDI [75].

As CDI surveillance is becoming mandatory and reportable to health regulatory authorities, laboratory test methods that are too sensitive may fall out of favor as these can lead to false positive results. The decision to select a specific diagnostic test for $C$. difficile may be driven by national health regulatory guidelines and requires input from all stake holders in a given health care facility. Although many FDA-approved commercial NAAT platforms are now available, there still remains some diagnostic challenges for CDI, as detection of $C$. difficile does not always correlate with disease. Finally, the diagnosis of CDI should be made clinically and confirmed by laboratory tests best suited to the local guidelines that can detect $C$. difficile reliably and accurately in acceptable stool specimens.

\section{References}

1. Lawson PA, Citron DM, Tyrrell KL, Finegold SM (2016) Reclassification of Clostridium difficile as Clostridioides difficile (Hall and O’Toole 1935) Prévot 1938. Anaerobe 40: 95-99.

2. Magill SS, Edwards JR, Bamberg W, Beldavs ZG, Dumyati G, Kainer MA, Lynfield R, Maloney M, McAllister-Hollod L, Nadle J, Ray SM, Thompson DL, Wilson LE, Fridkin SK; Emerging Infections Program Healthcare-Associated Infections and Antimicrobial Use Prevalence Survey Team (2014) Multistate point-prevalence survey of health careassociated infections. N Engl J Med 370: 1198-1208.

3. Peery AF, Dellon ES, Lund J, Crockett SD, McGowan CE, Bulsiewicz WJ, Gangarosa LM, Thiny MT, Stizenberg K, Morgan DR, Ringel Y, Kim HP, DiBonaventura MD, Carroll CF, Allen JK, Cook SF, Sandler RS, Kappelman MD, Shaheen NJ (2012) Burden of gastrointestinal disease in the United States: 2012 update. Gastroenterology 143: 1179-1187.e3.

4. Bauer MP, Notermans DW, van Benthem BHB, Brazier JS, Wilcox MH, Rupnik M, Monnet DL, van Dissel JT, Kuijper ED, ECDIS Study Group (2011) Clostridium difficile infection in Europe: a hospital-based survey. Lancet Lond Engl 377: 6373.

5. Lessa FC, Gould CV, McDonald LC (2012) Current status of Clostridium difficile infection epidemiology. Clin Infect Dis 55 Suppl 2: S65-70.

6. Burnham C-AD, Carroll KC (2013) Diagnosis of Clostridium difficile infection: an ongoing conundrum for clinicians and for clinical laboratories. Clin Microbiol Rev 26: 604-630.

7. Taur Y, Pamer EG (2014) Harnessing microbiota to kill a pathogen: Fixing the microbiota to treat Clostridium difficile infections. Nat Med 20: 246-247.

8. Schubert AM, Rogers MAM, Ring C, Mogle J, Petrosino JP, Young VB, Aronoff DM, Scloss PD (2014) Microbiome data distinguish patients with Clostridium difficile infection and non-C. difficile-associated diarrhea from healthy controls. mBio 5: e01021-01014.

9. Petrof EO, Gloor GB, Vanner SJ, Weese SJ, Carter D, Daigneault MC, Brown EM, Schroeter K, Allen-Vercoe E (2013) Stool substitute transplant therapy for the eradication of Clostridium difficile infection: "RePOOPulating" the gut. Microbiome 1: 3 .

10. Youngster I, Russell GH, Pindar C, Ziv-Baran T, Sauk J, Hohmann EL (2014) Oral, capsulized, frozen fecal microbiota transplantation for relapsing Clostridium difficile infection. JAMA 312: 1772-1778.

11. van Nood E, Vrieze A, Nieuwdorp M, Fuentes S, Zoetendal EG, de Vos WM, Visser CE, Kuijper EJ, Bartelsman JF, Tijssen JG, Speelman P, Dijkgraaf MG, Keller JJ (2013) Duodenal infusion of donor feces for recurrent Clostridium difficile. N Engl J Med 368: 407-415.

12. Kassam Z, Lee CH, Yuan Y, Hunt RH (2013) Fecal microbiota transplantation for Clostridium difficile infection: systematic review and meta-analysis. Am J Gastroenterol 108: 500-508.

13. Caroff DA, Edelstein PH, Hamilton K, Pegues DA, CDC Prevention Epicenters Program (2014) The Bristol stool scale and its relationship to Clostridium difficile infection. J Clin Microbiol 52: 3437-3439.

14. Lyerly DM, Krivan HC, Wilkins TD (1988) Clostridium difficile: its disease and toxins. Clin Microbiol Rev 1: 1-18.

15. Cohen SH, Gerding DN, Johnson S, Kelly CP, Loo VG, McDonald LC, Pepin J, Wilcox MH; Society for Healthcare Epidemiology of America; Infectious Diseases Society of 
America (2010) Clinical practice guidelines for Clostridium difficile infection in adults: 2010 update by the society for healthcare epidemiology of America (SHEA) and the infectious diseases society of America (IDSA). Infect Control Hosp Epidemiol 31: 431-455.

16. Planche T, Wilcox M (2011) Reference assays for Clostridium difficile infection: one or two gold standards? J Clin Pathol 64: $1-5$.

17. Hink T, Burnham CA, Dubberke ER (2013) A systematic evaluation of methods to optimize culture-based recovery of Clostridium difficile from stool specimens. Anaerobe 19: 3943.

18. Wren M (2010) Clostridium difficile isolation and culture techniques. Methods Mol Biol 646: 39-52.

19. Lyerly DM, Sullivan NM, Wilkins TD (1983) Enzyme-linked immunosorbent assay for Clostridium difficile toxin A. J Clin Microbiol 17: 72-78.

20. Kuehne SA, Cartman ST, Heap JT, Kelly ML, Cockayne A, Minton NP (2010) The role of toxin A and toxin B in Clostridium difficile infection. Nature 467: 711-713.

21. Quinn CD, Sefers SE, Babiker W, He Y, Alcabasa R, Stratton CW, Carroll KC, Tang Y (2010) C. Diff Quik Chek complete enzyme immunoassay provides a reliable first-line method for detection of Clostridium difficile in stool specimens. J Clin Microbiol 48: 603-605.

22. Novak-Weekley SM, Marlowe EM, Miller JM, Cumpio J, Nomura JH, Vance PH, Weissfeld A (2010) Clostridium difficile testing in the clinical laboratory by use of multiple testing algorithms. J Clin Microbiol 48: 889-893.

23. Dubberke ER, Han Z, Bobo L, Hink T, Lawrence B, Copper S, Hoppe-Bauer J, Burnham CD, Dunne Jr WM (2011) Impact of clinical symptoms on interpretation of diagnostic assays for Clostridium difficile infections. J Clin Microbiol 49: 28872893.

24. Carroll KC (2011) Tests for the diagnosis of Clostridium difficile infection: the next generation. Anaerobe 17: 170-174.

25. Crobach MJT, Dekkers OM, Wilcox MH, Kuijper EJ (2009) European Society of Clinical Microbiology and Infectious Diseases (ESCMID): data review and recommendations for diagnosing Clostridium difficile-infection (CDI). Clin Microbiol Infect 15: 1053-1066.

26. Goldenberg SD, Cliff PR, Smith S, Milner M, French GL (2010) Two-step glutamate dehydrogenase antigen real-time polymerase chain reaction assay for detection of toxigenic Clostridium difficile. J Hosp Infect 74: 48-54.

27. Tenover FC, Novak-Weekley S, Woods CW, Peterson LR, Davis T, Schreckenberger P, Fang FC, Dascal A, Gerding DN, Nomura JH, Goering RV, Akerlund T, Weissfeld AS, Baron EJ, Wong E, Marlowe EM, Whitmore J, Persing DH (2010) Impact of strain type on detection of toxigenic Clostridium difficile: comparison of molecular diagnostic and enzyme immunoassay approaches. J Clin Microbiol 48: 3719-3724.

28. Carman RJ, Wickham KN, Chen L, Lawrence AM, Boone JH, Wilkins TD, Kerkering TM, Lyerly DM (2012) Glutamate dehydrogenase is highly conserved among Clostridium difficile ribotypes. J Clin Microbiol 50: 1425-1426.

29. Goldenberg SD, French GL (2011) Diagnostic testing for Clostridium difficile: a comprehensive survey of laboratories in England. J Hosp Infect 79: 4-7.

30. Selvaraju SB, Gripka M, Estes K, Nguyen A, Jackson MA, Selvarangan R (2011) Detection of toxigenic Clostridium difficile in pediatric stool samples: an evaluation of Quik Check Complete Antigen assay, BD GeneOhm Cdiff PCR, and
ProGastro Cd PCR assays. Diagn Microbiol Infect Dis 71: 224-229.

31. Senok AC, Aldosari KM, Alowaisheq RA, Abid OA, Alsuhaibani KA, Khan MA, Somily AM (2017) Detection of Clostridium difficile antigen and toxin in stool specimens: Comparison of the $C$. difficile quik chek complete enzyme immunoassay and GeneXpert C. difficile polymerase chain reaction assay. Saudi J Gastroenterol 23: 259-262.

32. Vasoo S, Stevens J, Portillo L, Barza R, Schejbal D, Wu MM, Chancey C, Singh K (2014) Cost-effectiveness of a modified two-step algorithm using a combined glutamate dehydrogenase/toxin enzyme immunoassay and real-time PCR for the diagnosis of Clostridium difficile infection. J Microbiol Immunol Infect 47: 75-78.

33. Wren B, Clayton C, Tabaqchali S (1990) Rapid identification of toxigenic Clostridium difficile by polymerase chain reaction. Lancet Lond Engl 335: 423.

34. Kato N, Ou CY, Kato H, Bartley SL, Brown VK, Dowell Jr VR, Ueno K, (1991) Identification of toxigenic Clostridium difficile by the polymerase chain reaction. J Clin Microbiol 29: 33-37.

35. Gumerlock PH, Tang YJ, Weiss JB, Silva J (1993) Specific detection of toxigenic strains of Clostridium difficile in stool specimens. J Clin Microbiol 31: 507-511.

36. Dalpke AH, Hofko M, Zorn M, Zimmermann S (2013) Evaluation of the fully automated BD MAX Cdiff and Xpert C. difficile assays for direct detection of Clostridium difficile in stool specimens. J Clin Microbiol 51: 1906-1908.

37. Pancholi P, Kelly C, Raczkowski M, Balada-Llasat JM (2012) Detection of toxigenic Clostridium difficile: comparison of the cell culture neutralization, Xpert C. difficile, Xpert C. difficile/Epi, and Illumigene C. difficile assays. J Clin Microbiol 50: 1331-1335.

38. Goldenberg SD, Bisnauthsing KN, Patel A, Postulka A, Wyncoll D, Schiff R, French GL (2014) Point-of-Care Testing for Clostridium difficile Infection: A Real-World Feasibility Study of a Rapid Molecular Test in Two Hospital Settings. Infect Dis Ther 3: 295-306.

39. Stellrecht KA, Espino AA, Maceira VP, Nattanmai SM, Butt SA, Wroblewski D, Hannett GE, Musser KA (2014) Premarket evaluations of the IMDx C. difficile for Abbott m2000 Assay and the BD Max Cdiff Assay. J Clin Microbiol 52: 1423-1428.

40. Carroll KC, Buchan BW, Tan S, Stamper PD, Riebe KM, Pancholi P, Kelly C, Rao A, Fader R, Cavagnolo R, Watson W, Goering RV, Trevino EA, Weissfeld AS, Ledeboer NA (2013) Multicenter evaluation of the Verigene Clostridium difficile nucleic acid assay. J Clin Microbiol 51: 4120-4125.

41. Deak E, Miller SA, Humphries RM (2014) Comparison of Illumigene, Simplexa, and AmpliVue Clostridium difficile molecular assays for diagnosis of $\mathrm{C}$. difficile infection. J Clin Microbiol 52: 960-963.

42. Buchan BW, Mackey TA, Daly JA, Alger G, Denys GA, Peterson LR, Kehl SC, Ledeboer NA (2012) Multicenter clinical evaluation of the portrait toxigenic C. difficile assay for detection of toxigenic Clostridium difficile strains in clinical stool specimens. J Clin Microbiol 50: 3932-3936.

43. Notomi T, Okayama H, Masubuchi H, Yonekawa T, Watanabe $\mathrm{K}$, Amino N, Hase T (2000) Loop-mediated isothermal amplification of DNA. Nucleic Acids Res 28: E63.

44. Nagamine K, Hase T, Notomi T (2002) Accelerated reaction by loop-mediated isothermal amplification using loop primers. Mol Cell Probes 16: 223-229. 
45. Rupnik M, Janezic S (2016) An Update on Clostridium difficile Toxinotyping. J Clin Microbiol 54: 13-18.

46. Moukhaiber R, Araj GF, Kissoyan KAB, Cheaito KA, Matar GM (2015) Prevalence of Clostridium difficile toxinotypes in infected patients at a tertiary care center in Lebanon. J Infect Dev Ctries 9: 732-735. doi: 10.3855/jidc.6585.

47. Gerding DN, Johnson S, Rupnik M, Aktories K (2014) Clostridium difficile binary toxin CDT: mechanism, epidemiology, and potential clinical importance. Gut Microbes 5: 15-27.

48. Yoo J, Park J, Lee HK, Yu JK, Lee GD, Park KG, Oak HC, Park Y (2019) Comparative Evaluation of Seegene Allplex Gastrointestinal, Luminex xTAG Gastrointestinal Pathogen Panel, and BD MAX Enteric Assays for Detection of Gastrointestinal Pathogens in Clinical Stool Specimens. Arch Pathol Lab Med 143: 999-1005.

49. Shin B, Mun SJ, Yoo SJ, Kuak EY (2012) Comparison of BD GeneOhm Cdiff and Seegene Seeplex ACE PCR assays using toxigenic Clostridium difficile culture for direct detection of tcdB from stool specimens. J Clin Microbiol 50: 3765-3767.

50. Drancourt M, Michel-Lepage A, Boyer S, Raoult D (2016) The Point-of-Care Laboratory in Clinical Microbiology. Clin Microbiol Rev 29: 429-447.

51. Cohen DM, Russo ME, Jaggi P, Kline J, Gluckman W, Parekh A (2015) Multicenter Clinical Evaluation of the Novel Alere i Strep A Isothermal Nucleic Acid Amplification Test. J Clin Microbiol 53: 2258-2261.

52. Weber NC, Klepser ME, Akers JM, Klepser DG, Adams AJ (2016) Use of CLIA-waived point-of-care tests for infectious diseases in community pharmacies in the United States. Expert Rev Mol Diagn 16: 253-264.

53. Bissonnette L, Bergeron MG (2016) The GenePOC Platform, a Rational Solution for Extreme Point-of-Care Testing. Micromachines 7.

54. Stubbs SL, Brazier JS, O’Neill GL, Duerden BI (1999) PCR targeted to the 16S-23S rRNA gene intergenic spacer region of Clostridium difficile and construction of a library consisting of 116 different PCR ribotypes. J Clin Microbiol 37: 461-463.

55. Bidet P, Barbut F, Lalande V, Burghoffer B, Petit JC (1999) Development of a new PCR-ribotyping method for Clostridium difficile based on ribosomal RNA gene sequencing. FEMS Microbiol Lett 175: 261-266.

56. Manzoor SE, Tanner HE, Marriott CL, Brazier JS, Hardy KJ, Platt S, Hawkey PM, (2011) Extended multilocus variablenumber tandem-repeat analysis of Clostridium difficile correlates exactly with ribotyping and enables identification of hospital transmission. J Clin Microbiol 49: 3523-3530.

57. Indra $A$, Huhulescu $S$, Schneeweis $M$, Hasenberger $P$, Kernbichler S, Fiedler A, Wewelka G, Allerberger F, Kuijper EJ (2008) Characterization of Clostridium difficile isolates using capillary gel electrophoresis-based PCR ribotyping. J Med Microbiol 57: 1377-1382.

58. Wilcox MH, Shetty N, Fawley WN, Shemko M, Coen P, Birtles A, Cairns M, Curran MD, Dodgson KJ, Green SM, Hardy KJ, Hawkey PM, Magee JG, Sails AD, Wren MW (2012) Changing epidemiology of Clostridium difficile infection following the introduction of a national ribotypingbased surveillance scheme in England. Clin Infect Dis 55: 1056-1063.

59. Davies KA, Ashwin H, Longshaw CM, Burns DA, Davis GL, Wilcox MH, EUCLID Study Group (2016) Diversity of Clostridium difficile PCR ribotypes in Europe: results from the European, multicentre, prospective, biannual, point-prevalence study of Clostridium difficile infection in hospitalised patients with diarrhoea (EUCLID), 2012 and 2013. Euro Surveill Bull 21;21(29).

60. Hensgens MPM, Keessen EC, Squire MM, Riley TV, Koene MGJ, de Boer E, Lipman LJA, Kuijper EJ (2012) Clostridium difficile infection in the community: a zoonotic disease? Clin Microbiol Infect 18: 635-645.

61. Lessa FC, Mu Y, Bamberg WM, Beldavs ZG, Dumyati GK, Dunn JR, Farley MM, Holzbauer SM, Meek JI, Phipps EC, Wilson LE, Winston LG, Cohen JA, Limbago BM, Fridkin SK, Gerding DN, McDonald LC (2015) Burden of Clostridium difficile infection in the United States. N Engl J Med 372: 825834.

62. Kristjánsson M, Samore MH, Gerding DN, DeGirolami PC, Bettin KM, Karchmer AW, Arbeit RD (1994) Comparison of restriction endonuclease analysis, ribotyping, and pulsed-field gel electrophoresis for molecular differentiation of Clostridium difficile strains. J Clin Microbiol 32: 1963-1969.

63. Killgore G, Thompson A, Johnson S, Brazier J, Kuijper E, Pepin J, Frost EH, Savelkoul P, Nicholson B, van den Berg RJ, Kato H, Sambol SP, Zukowski W, Woods C, Limbago B, Gerding DN, McDonald LC (2008) Comparison of seven techniques for typing international epidemic strains of Clostridium difficile: restriction endonuclease analysis, pulsedfield gel electrophoresis, PCR-ribotyping, multilocus sequence typing, multilocus variable-number tandem-repeat analysis, amplified fragment length polymorphism, and surface layer protein A gene sequence typing. J Clin Microbiol 46: 431-437.

64. Marsh JW, O'Leary MM, Shutt KA, Pasculle AW, Johnson S, Gerding DN, Muto CA, Harrison LH (2006) Multilocus variable-number tandem-repeat analysis for investigation of Clostridium difficile transmission in Hospitals. J Clin Microbiol 44: 2558-2566.

65. Lemee L, Dhalluin A, Pestel-Caron M, Lemeland JF, Pons JL (2004) Multilocus sequence typing analysis of human and animal Clostridium difficile isolates of various toxigenic types. J Clin Microbiol 42: 2609-2617.

66. Griffiths D, Fawley W, Kachrimanidou M, Bowden R, Crook DW, Fung R, Golubchik T, Harding RM, Jeffery KJ, Jolley KA, Kirton R, Peto TE, Rees G, Stoesser N, Vaughn A, Walker AS, Young BC, Wilcox M, Dingle KE (2010) Multilocus sequence typing of Clostridium difficile. J Clin Microbiol 48: 770-778.

67. Larsen MV, Cosentino S, Rasmussen S, Friis C, Hasman H, Marvig RL, Jelsbak L Sicheritz-Ponten T, Ussery DW, Aarestrup FM, Lund O (2012) Multilocus sequence typing of total-genome-sequenced bacteria. J Clin Microbiol 50: 13551361.

68. Reuter S, Ellington MJ, Cartwright EJP, Köser CU, Török ME, Gouliouris T, Harris SR, Brown NM, Holden MT, Quail M, Parkhill J, Smith GP, Bentley SD, Peacock SJ (2013) Rapid bacterial whole-genome sequencing to enhance diagnostic and public health microbiology. JAMA Intern Med 173: 13971404.

69. Chan JZ-M, Pallen MJ, Oppenheim B, Constantinidou C (2012) Genome sequencing in clinical microbiology. Nat Biotechnol 30: 1068-1071.

70. Kong LY, Eyre DW, Corbeil J, Raymond F, Walker AS, Wilcox MH, Crook DW, Michaud S, Toye B, Frost E, Dendukuri N, Schiller I, Bourgault AM, Dascal A, Oughton M, Longtin Y, Poirier L, Brassard P, Turgeon N, Gilca R, Loo VG (2019) Clostridium difficile: Investigating Transmission 
Patterns Between Infected and Colonized Patients Using Whole Genome Sequencing. Clin Infect Dis 68: 204-209.

71. Mac Aogáin M, Moloney G, Kilkenny S, Kelleher M, Kelleghan M, Boyle B, Rogers TR (2015) Whole-genome sequencing improves discrimination of relapse from reinfection and identifies transmission events among patients with recurrent Clostridium difficile infections. J Hosp Infect 90: 108-116.

72. Balloux F, Brønstad Brynildsrud O, van Dorp L, Shaw LP, Chen H, Harris KA, Wang H, Eldholm V (2018) From Theory to Practice: Translating Whole-Genome Sequencing (WGS) into the Clinic. Trends Microbiol 26: 1035-1048.

73. Gateau C, Couturier J, Coia J, Barbut F (2018) How to: diagnose infection caused by Clostridium difficile. Clin Microbiol Infect 24: 463-468.

74. Halstead DC, Abid J, Sloan L, Meza D, Ramsey-Walker D, Hata DJ (2016) A multi-laboratory comparison of two molecular methods for the detection of toxigenic Clostridium difficile. J Infect Dev Ctries 10: 62-67. doi: 10.3855/jidc.6634.
75. Choucair J, Waked R, Haddad E, Chedid M, Chehata N, Saliba G, Dahboul H (2020) Clostridioides difficile infections: Epidemiology, correlations and treatment in a Lebanese cohort with use of ATLAS scoring. J Infect Dev Ctries 14: 14611465. doi: $10.3855 /$ jidc. 13189 .

\section{Corresponding author}

Ali Somily, MBBS, FRCPC, D(ABMM), FCCM

Department of Pathology and Laboratory Medicine

College of Medicine, King Saud University and King Saud

University Medical City, PO Box 2925, Riyadh 11461, Kingdom of Saudi Arabia.

Phone: +96611-467-2640; +96611467-1010

Fax:+96611-467-2462

Email: somily@ksu.edu.sa; ali.somily@gmail.com

Conflict of interests: No conflict of interests is declared. 